\title{
BitTorrent and fountain codes: friends or foes?
}

Salvatore Spoto, Rossano Gaeta, Marco Grangetto, Matteo Sereno

Dipartimento di informatica

Università di Torino 
Peer to peer paradigm has a huge diffusion nowadays [1]

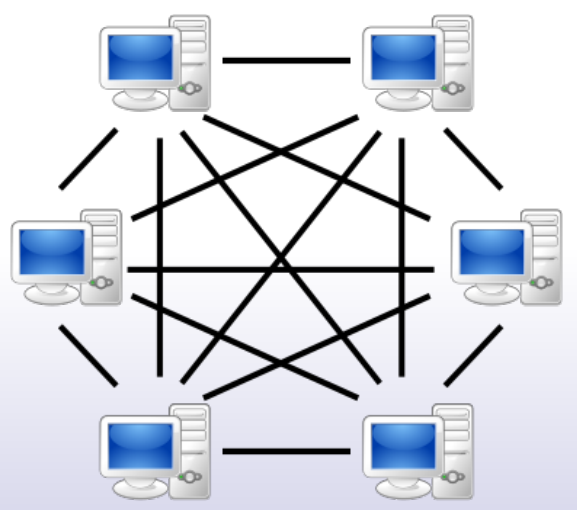

It can be employed for a lot of different applications:

- File sharing: BitTorrent [2][3] , eDonkey, eMule, DC++

- Video Streaming : SopCast, PPLive, PPStream

- Distribuited portals: Osiris

- Others: Skype, Sciencenet, Spotify

BitTorrent is doubtless the reference architecture for file sharing 
BitTorrent adopts a multi-part download scheme:

File's pieces

\begin{tabular}{|l|l|l|l||l||l|}
\hline p1 & $\mathrm{p} 2$ & $\mathrm{p} 3$ & $\mathrm{p} 4$ & $\mathrm{p} 5$ & $\mathrm{p} 6$ \\
\hline
\end{tabular}

- The file is divided into pieces, each piece into blocks.

- Peers are synchronized between them at piece level.

- Each client knows what pieces have been completely downloaded from his neighbors.

- Peers exchange between them piece's blocks 


\section{BitTorrent protocol 2: rarest first and tit for tat}

Main BitTorrent's strategies:

- Tit-for-Tat: assures reciprocity between downloading and uploading rates.

- Rarest first: assures a fair distribution of file's pieces

Note that only complete pieces can be shared and distributed

Many research works claim that BitTorrent has near-optimal performance [4] [5] [6] 
Some phenomena can cripple BitTorrent performance:

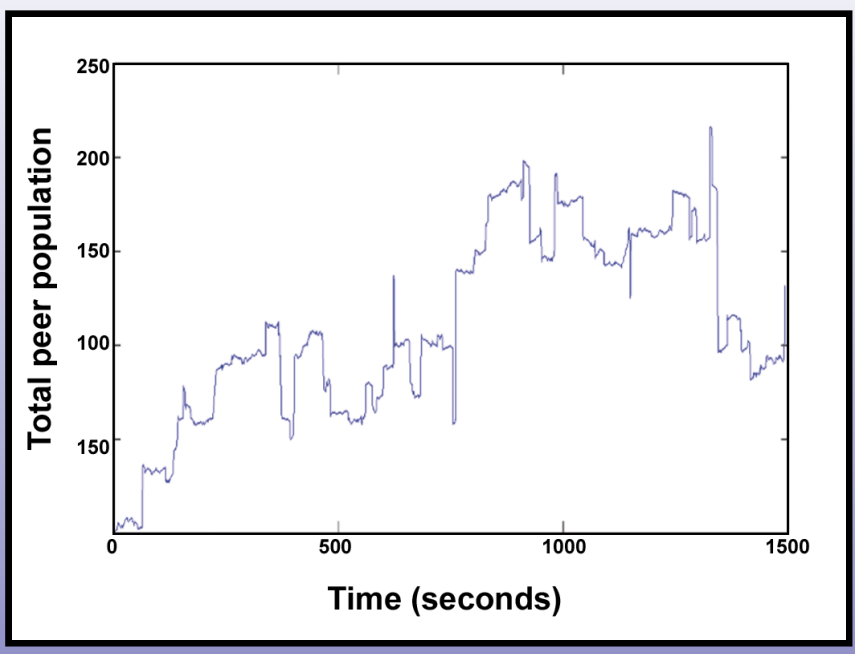

Example of simulated population's dynamics in a network affected by flash crowd and high Churn rates.
- Flash crowd: many peer join or leave the network at the same time

- High churn rates: peers join and leave the network at high rates

- Many peer leave the overlay network at the same time 
LT codes [7][8] are a class a fountain codes that use simple XOR operations to encode and decode the message. The decoding process has a certain overhead $\boldsymbol{\varepsilon}$ : the recipient needs to receive a number of packets that exceed in percentage the original size of the message by a certain amount.

If $\mathbf{k}$ is the original number of non coded blocks, the decoding process succeeds when $\mathbf{k}(1+\boldsymbol{\varepsilon})$ packets are received.

\section{Benefits}

- Avoid content reconciliation

- A peer can share partially downloaded file parts

- File parts can be download concurrently from different peers

\section{Drawbacks}

- Decoding overhead

- Encoder/decoder complexity
Uncoded protocol

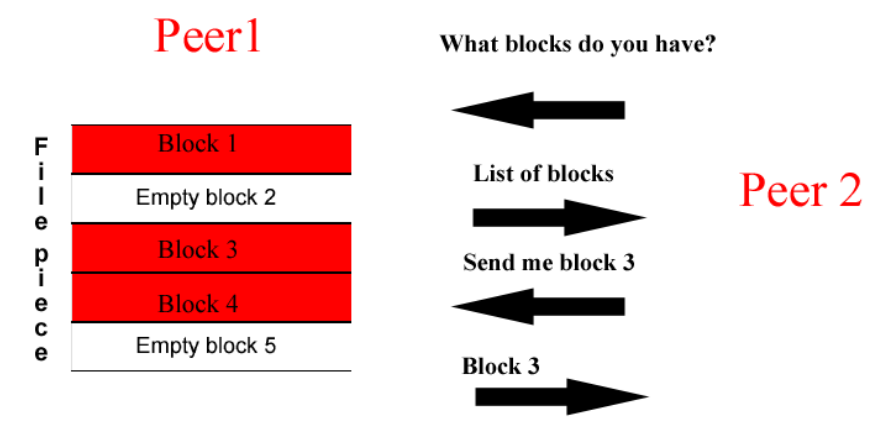

But block 3 is only $16 \mathrm{~KB}$ : is not convenient share partial blocks, too much signalling overhead

Using LT codes

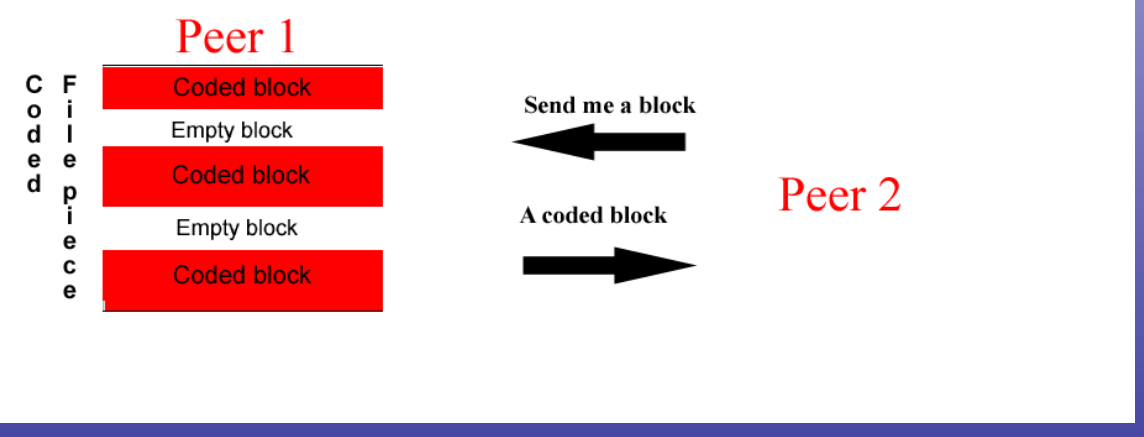

Coded blocks can be sended without content reconciliation 
Summary of protocol modifications:

- Use LT codes to code file's pieces. The file's piece subdivision is different in accordance with the requirements of encoding. The decoding overhead is about $10 \%$

- Share partial file's piece

- A piece can be downloaded from more peers at same time without content reconciliation

- The protocol requests first the rarest complete piece. If there are not complete pieces available, a peer requests the rarest partial piece

- If a piece is fully decoded a peer sends new coded blocks

We use General purpose simulator GPS[9] simulator in order to test our modified protocol 


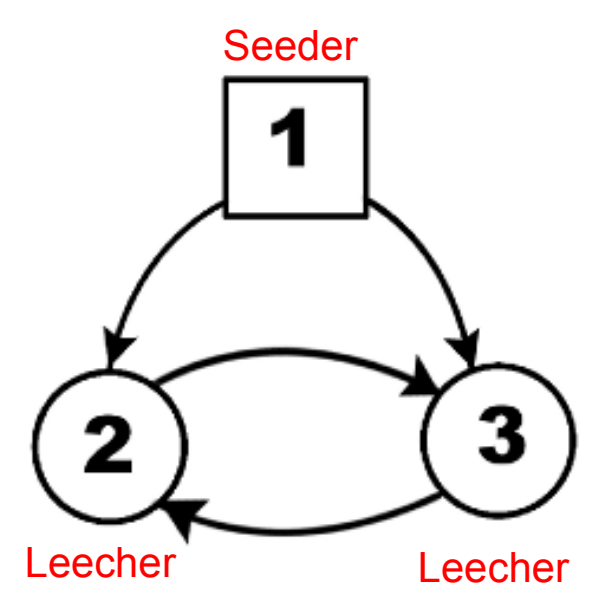

A simple topology with a seeder and two leechers

DOWNLOADING TIMES AND ACHIEVABLE BITRATE OBSERVED ON A SIMPLE TOPOLOGY OF THREE PEERS.

\begin{tabular}{l||c||c||c||c||c||}
\hline File size & BTd $^{1}$ & $\mathbf{L T d}^{2}$ & BTb $^{\mathbf{1}}$ & $\mathbf{L T b}^{\mathbf{2}}$ & Gain (\%) \\
\hline \hline 1 MBytes & $38 \mathrm{~s}$ & $35 \mathrm{~s}$ & $210 \mathrm{Kbps}$ & $228 \mathrm{Kbps}$ & $8 \%$ \\
\hline \hline 2 MBytes & $50 \mathrm{~s}$ & $48 \mathrm{~s}$ & $320 \mathrm{Kbps}$ & $333 \mathrm{Kbps}$ & $4 \%$ \\
\hline \hline 4 MBytes & $77 \mathrm{~s}$ & $75 \mathrm{~s}$ & $415 \mathrm{Kbps}$ & $426 \mathrm{Kbps}$ & $2.6 \%$ \\
\hline${ }^{1}$ the columns BTd and BTb show respectively the download times and \\
achievable bitrate of the standart BitTorrent protocol \\
${ }^{2}$ the columns LTd and LTb show respectively the download times and \\
achievable bitrate of the modified protocol that use the LT codes
\end{tabular}


Single flash crowd scenario set up:

- Time 0: a flash crowd of 50 peers occurs

- Time 50: 20 random selected peers leave the network, regardless of the state of the download

DOWNLOADING TIMES AND ACHIEVABLE BITRATE OBSERVED IN A SINGLE FLASH CROWD SCENARIO.

\begin{tabular}{c||c||c||c||c||c||c}
\hline File size & PS $^{\mathbf{1}}$ & BTd $^{\mathbf{2}}$ & LTd $^{\mathbf{3}}$ & $\mathbf{B T b}^{\mathbf{2}}$ & $\mathbf{L T b}^{\mathbf{3}}$ & Gain \\
\hline \hline 1 MByte & $\mathrm{S}$ & $53.1 \mathrm{~s}$ & $18.1 \mathrm{~s}$ & $151 \mathrm{Kbps}$ & $441 \mathrm{Kbps}$ & $66 \%$ \\
\hline \hline 1 MByte & $\mathrm{M}$ & $23.9 \mathrm{~s}$ & $17.1 \mathrm{~s}$ & $334 \mathrm{Kbps}$ & $467 \mathrm{Kbps}$ & $26 \%$ \\
\hline
\end{tabular}

${ }^{1}$ the file subdivision, single $(\mathbf{S})$ or multiple $(\mathbf{M})$ pieces

2 the columns BTd and BTb show respectively the download times and achievable bitrate of the standart BitTorrent protocol

${ }^{3}$ the columns LTd and LTb show respectively the download times and achievable bitrate of the modified protocol that use the LT codes 

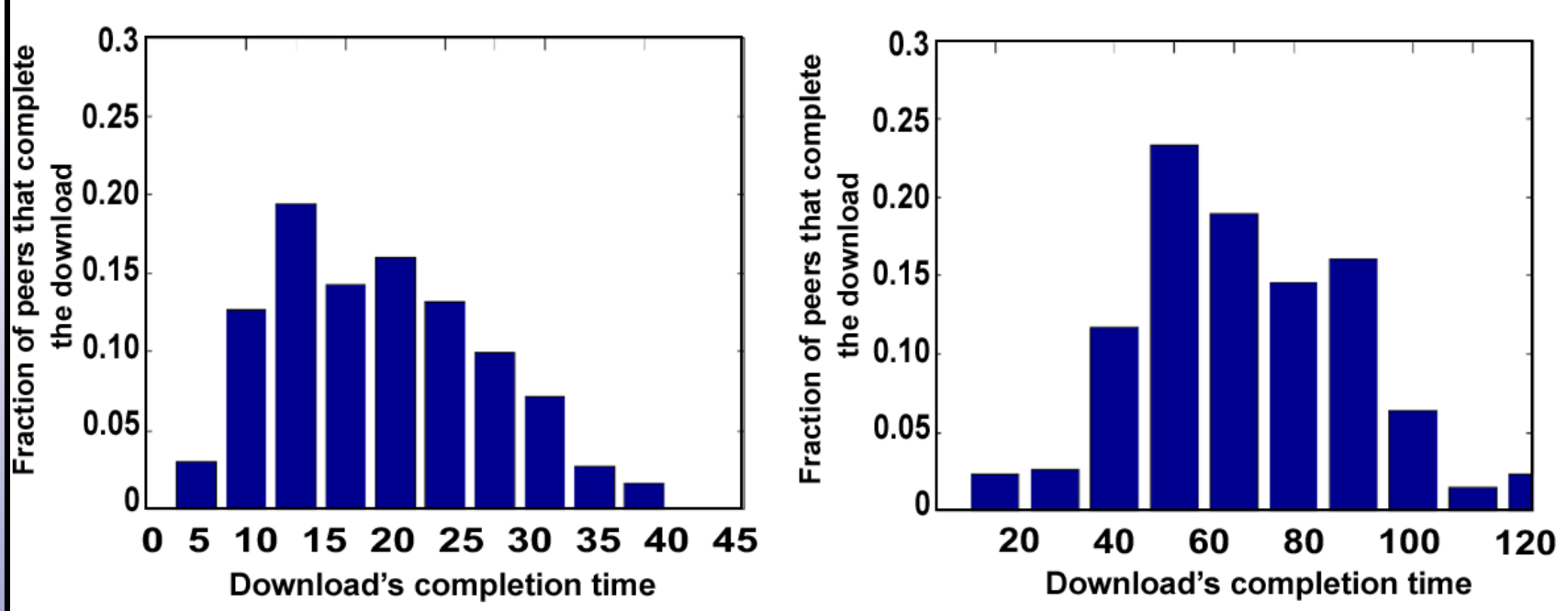

Comparison of downloading time distributions between original protocol and the modified one 


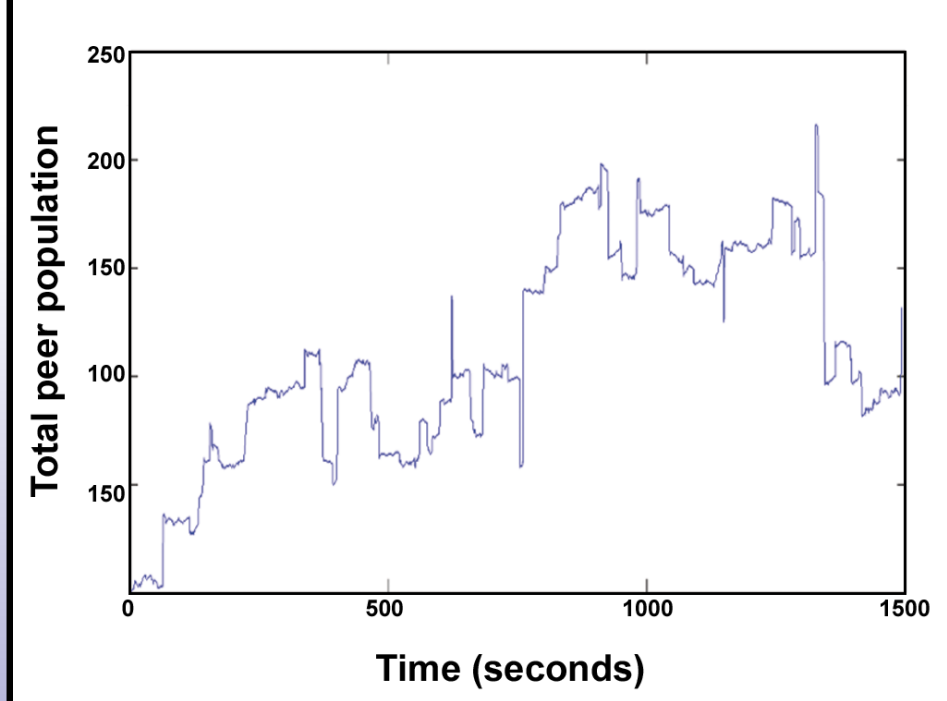

During the simulation multiple flash crowd and massive departure of peer occur

Example of simulated population's dynamics in a network affected by flash crowd and high Churn rates.

DOWNLOADING TIMES AND ACHIEVABLE BITRATE OBSERVED IN A SCENARIO AFFECTED BY MULTIPLE FLASH CRWOD AND MASSIVE DEPARTURE OF PEERS.

\begin{tabular}{c||c||c||c||c||c}
\hline File size & BTd $^{\mathbf{1}}$ & LTd $^{\mathbf{2}}$ & BTb $^{\mathbf{1}}$ & LTb $^{\mathbf{2}}$ & Gain \\
\hline \hline $4 \mathrm{MBytes}$ & $65 \mathrm{~s}$ & $56 \mathrm{~s}$ & $492 \mathrm{Kbps}$ & $571 \mathrm{Kbps}$ & $14 \%$ \\
\hline \hline $8 \mathrm{MBytes}$ & $145 \mathrm{~s}$ & $129 \mathrm{~s}$ & $441 \mathrm{Kbps}$ & $496 \mathrm{Kbps}$ & $12 \%$ \\
\hline
\end{tabular}

${ }^{1}$ the columns BTd and BTb show respectively the download times and achievable bitrate of the standart BitTorrent protocol

2 the columns LTd and LTb show respectively the download times and achievable bitrate of the modified protocol that use the LT codes 
In this paper we proposed a novel modifications of the BitTorrent protocol by introducing the LT encoding Then we proved by simulations that better performance can be achieved considering file of small size and in adverse network conditions. In such situation our proposed protocol yields a gain that is above the $10 \%$ in all simulated scenarios, in spite of the decoding overhead that the LT codes introduce. 
Thank you for your kind attention 
[1] T. Karagiannis, A. Broido, M. Faloutsos, and K. C. Claffy, Transport layer identification of p2p traffic, in Proc. ACM IMC04, Taormina, Sicily, Italy, October 2004.

[2] http://www.bittorrent.com/

[3] Bitorrent protocol specification v1.0 http://wiki.theory.org/BitTorrentSpecification, June 2005

[4] A. Legout, G. Urvoy-Keller, P. Michiardi, Understanding bittorrent: An experimental perspective. Technical report, CNRS : FRE2660 EURECOM

[5] A. Legout, G. Urvoy-Keller, P. Michiardi, Rarest First and Choke Algorithms Are Enough, IMC '06: Proceedings of the 6th ACM SIGCOMM conference on Internet measurement, Rio de Janeriro, Brazil, 2006.

[6] A. Al-Hamra, A. Legout, and C. Barakat, Understanding the Properties of the BitTorrent Overlay, CoRR abs/0707.1820: (2007).

[7] M. Luby, LT codes, Foundations of Computer Science, 2002. Proceedings. The 43rd Annual IEEE Symposium on (2002).

[8] E.Hyytia, T. Tirronen, J. Virtamo Optimizing the Degree Distribution of LT Codes, in RESIM 2006, 6th International Workshop on Rare Event Simulation, 2006, Bamberg, Germany

[9] W. Yang, N. Abu-Ghazaleh, GPS: A General Peer-to-Peer Simulator and its Use for Modeling BitTorrent, Proceedings of 13th Annual Meeting of the IEEE International Symposium on Modeling, Analysis, and Simulation of Computer and Telecommunication Systems (MASCOTS

'05), Sept 27-29, 2005, Atlanta. 